\title{
RESEARCH
}

Open Access

\section{Gut bacterial ClpB-like gene function is associated with decreased body weight and a characteristic microbiota profile}

María Arnoriaga-Rodríguez 1,2,3,4 , Jordi Mayneris-Perxachs 1,2,3, Aurelijus Burokas5 ${ }^{5,6}$, Vicente Pérez-Brocal7,8, Andrés Moya ${ }^{7,8,9}$, Manuel Portero-Otin ${ }^{10}$, Wifredo Ricart ${ }^{1,2,3,4}$, Rafael Maldonado ${ }^{5,11}$ and José-Manuel Fernández-Real ${ }^{1,2,3,4^{*}}$

\begin{abstract}
Background: The chaperone $\mathrm{ClpB}$, a bacterial protein, is a conformational antigen-mimetic of a-melanocytestimulating hormone (a-MSH) implicated in body weight regulation in mice. We here investigated the potential associations of gut bacterial ClpB-like gene function with obesity status and gut microbiota in humans.

Results: Gut microbiota ClpB KEGG function was negatively associated with body mass index, waist circumference, and total fat mass (DEXA). The relative abundance (RA) of several phyla and families directly associated with ClpB was decreased in subjects with obesity. Specifically, the RA of Rikenellaceae, Clostridiaceae and not assigned Firmicutes were lower in subjects with obesity and positively associated with gut bacterial ClpB-like gene function (not assigned Firmicutes $(r=0.405, \mathrm{FDR}=2.93 \times 10-2)$, Rikenellaceae $(r=0.217, \mathrm{FDR}=0.031)$, and Clostridiaceae $(r=$ 0.239 , FDR $=0.017)$ ). The gut bacterial ClpB-like gene function was also linked to specific plasma metabolites (hippuric acid and 3-indolepropionic acid) and fecal lupeol. The a-MSH-like epitope similar to that of Escherichia coli ClpB was also identified in some sequences of those bacterial families.

After fecal transplantation from humans to mice, the families that more contributed to ClpB-like gene function in humans were also associated with ClpB-like gene function in mice after adjusting for the donor's body mass index (not assigned Firmicutes $(r=0.621, p=0.003)$, Prevotellaceae $\left(r=0.725, p=4.1 \times 10^{-7}\right)$, Rikenellaceae $(r=0.702, p=$ $\left.3.9 \times 10^{-4}\right)$, and Ruminococcaceae $\left.(r=0.526, p=0.014)\right)$. Clostridiaceae $(r=-0.445, p=0.038)$ and Prevotellaceae RA $(r=-0.479, p=0.024)$ and were also negatively associated with weight gain in mice. The absolute abundance (AA) of Prevotellaceae in mice was also positively associated with the gut bacterial ClpB-like gene function in mice. DESeq2 identified species of Prevotellaceae, both negatively associated with mice' weight gain and positively with gut bacterial ClpB-like gene function.
\end{abstract}

Conclusions: In summary, gut bacterial ClpB-like gene function is associated with obesity status, a specific gut microbiota composition and a plasma metabolomics profile in humans that could be partially transplanted to mice.

Keywords: Microbiome, Bacterial gene function, Body weight regulation, Obesity

\footnotetext{
* Correspondence: jmfreal@idibgi.org

'Department of Diabetes, Endocrinology and Nutrition, Dr. Josep Trueta University Hospital, Carretera de França s/n, 17007 Girona, Spain

${ }^{2}$ Nutrition, Eumetabolism and Health Group, Girona Biomedical Research Institute (IdibGi), Girona, Spain

Full list of author information is available at the end of the article
}

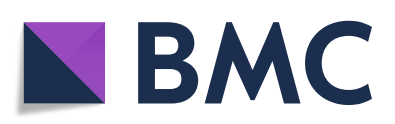

(- The Author(s). 2020 Open Access This article is licensed under a Creative Commons Attribution 4.0 International License, which permits use, sharing, adaptation, distribution and reproduction in any medium or format, as long as you give appropriate credit to the original author(s) and the source, provide a link to the Creative Commons licence, and indicate if changes were made. The images or other third party material in this article are included in the article's Creative Commons licence, unless indicated otherwise in a credit line to the material. If material is not included in the article's Creative Commons licence and your intended use is not permitted by statutory regulation or exceeds the permitted use, you will need to obtain permission directly from the copyright holder. To view a copy of this licence, visit http://creativecommons.org/licenses/by/4.0/ The Creative Commons Public Domain Dedication waiver (http://creativecommons.org/publicdomain/zero/1.0/) applies to the data made available in this article, unless otherwise stated in a credit line to the data. 


\section{Background}

Obesity is rapidly becoming a global epidemic and its management is a priority challenge to halting this tendency. The role of the microbiota in the pathophysiology of obesity [1] and related complications [2] is increasingly recognized. The microbiota-gut-brain axis has been associated with the regulation of metabolism, energy balance, adiposity, central appetite, and food reward [3, 4]. Gut microbiota could modulate intestinal satiety and hunger hormones such as glucagon-like peptide-1 (GLP1), peptide YY (PYY), and ghrelin [5] that impact on anorexigenic and orexigenic pathways, and also proopiomelanocortin (POMC) and neuropeptide Y (NPY)/agoutirelated protein $(\mathrm{AgRP})$ neurons, finally integrated in the hypothalamus [6].

Host energy homeostasis could be regulated by the bacterial production of metabolites and neurotransmitters or even by energy harvesting of their own bacterial metabolism [7, 8]. Recently, it has also been observed that bacterial proteins which directly act in the brain via vagal stimulation or indirectly through immuneneuroendocrine mechanisms have an important role in this process [7, 9]. One of these bacterial proteins, the caseinolytic peptidase $\mathrm{B}$ protein homolog $(\mathrm{ClpB})$, has been identified as a conformational antigen-mimetic of $\alpha$-melanocyte-stimulating hormone $(\alpha-\mathrm{MSH})$ [10]. The $\alpha-\mathrm{MSH}$ is an amino-acid derived from POMC that activates the melanocortin-4 receptor (MC4R) expressed in the hypothalamic paraventricular nucleus promoting the anorexigenic pathway and therefore, regulating satiety, energy, blood pressure, and growth [11].

Tennoune et al. found that ClpB-immunized mice produced anti-ClpB IgG cross-reactive with $\alpha-\mathrm{MSH}$, influencing food intake and body weight. In addition, these authors reported increased plasma levels of an antibody anti-ClpB in patients with anorexia nervosa, bulimia, and binge-eating disorder [10]. On the other hand, Breton et al. described how regular nutrient provision stabilized exponential growth of Escherichia coli, with the stationary phase occurring 20 min after nutrient supply [9]. ClpB was upregulated in the E. coli stationary phase and plasma $\mathrm{ClpB}$ was proportional to $\mathrm{ClpB}$ DNA in feces, stimulating the firing rate of hypothalamic POMC neurons [9]. Furthermore, the administration of a probiotic, Hafnia alvei, a ClpB-producing bacterium, decreased fat mass and food intake in $o b / o b$ and after a high-fat diet in mice [12].

To our knowledge, there is little evidence assessing ClpB gene function in subjects with obesity. Current information points to a lower gene richness in individuals with obesity and a negative association with body mass index of bacteria belonging to genera of Enterobacter, Klebsiella, and Hafnia in sillico analysis using the MetaHIT database [12]. Therefore, our main aim was to evaluate gut bacterial ClpB-like gene function in subjects with obesity compared to controls and assess the potential role of the microbiota composition and microbialderived compounds in the modulation of body weight.

\section{Results}

Gut bacterial ClpB-like gene function is associated with decreased body weight in humans

A consecutive series of 131 subjects, 76 of them with obesity and their respective paired by sex and age controls, was studied (Table 1). The detection of the gut bacterial ClpB-like gene function assessed using shotgun metagenomic analysis of fecal microbiota using the KEGG annotation, K03695 (subcategory (sc): aging/protein families: genetic information processing; pathway (p): longevity regulating pathway-multiple species [PATH:ko04213]/chaperones and folding catalysts [BR: ko03110]; annotation description (ad): ClpB ATPdependent Clp protease ATP-binding subunit ClpB; and annotation (a): K03695). This function was significantly lower in subjects with obesity (Fig. 1a; Table 1). In addition, this ClpB-like gene function was negatively associated with body mass index (Fig. 1b), waist circumference (Fig. 1c), and total fat mass (Fig. 1d). Other KEGG functions were also negatively associated with body mass index but not so strongly, such as K01358 $(r=-0.277, p$ $=0.001$; sc: cell growth and death/aging/protein families: metabolism; p: cell cycle-Caulobacter [PATH: ko04112]/longevity regulating pathway-worm [PATH: ko04212]/peptidases [BR:ko01002]; ad: ClpP, CLPP ATP-dependent Clp protease, protease subunit [EC: 3.4.21.92]; a: K01358) and K01419 $(r=-0.276, p=$ 0.001 , sc: protein families: metabolism; p: peptidases [BR:ko01002]; ad: hslV, clpQ ATP-dependent HslUV protease, peptidase subunit HslV [EC:3.4.25.2]; and a: K01419).

As diet is a well-known modifier of bacterial ecosystems, we explored which dietary components, by focusing on macronutrients and fiber, were linked to gut bacterial ClpB-like gene function (K03695). As a crude measure, total energy intake was negatively associated with ClpB-like gene function $(r=-0.189, p=0.041)$. After adjusting for total energy intake and body weight, gut bacterial ClpB-like function was directly associated with proteins $(r=0.200, p=0.032)$, carbohydrates $(r=$ $0.224, p=0.015)$, lipids $(r=0.303, p=0.001)$, and fiber intake $(r=0.193, p=0.037)$. Nevertheless, in multiple linear regression models, with gut bacterial ClpB-like gene function (K03695) as dependent variable and body mass index, sex, age, total energy intake, and each macronutrient or fiber, as independent variables, only body mass index (e.g., model with proteins, $\beta=-0.345$, $p=4.3 \times 10^{-4}$ ) remained significantly associated with gut bacterial ClpB-like gene function. 
Table 1 Clinical characteristics of the human samples

\begin{tabular}{|c|c|c|c|}
\hline & Without obesity & With obesity & \\
\hline & $(n=55)$ & $(n=76)$ & $p$ value \\
\hline Females $n(\%)$ & $37(67.3)$ & $52(68.4)$ & 0.519 \\
\hline Age (years) & $53.7[17.4]$ & $48.6[15.7]$ & 0.188 \\
\hline BMI $\left(\mathrm{kg} / \mathrm{m}^{2}\right)$ & $24.9(2.5)$ & $43.2(6.8)$ & $<0.001$ \\
\hline Waist (cm) & $89.7(9.6)$ & $126.2(14.1)$ & $<0.001$ \\
\hline Fat total (\%) & $32.5(7.5)$ & $49.6(5.6)$ & $<0.001$ \\
\hline Android fat (\%) & $32.6(10.5)$ & $56.5(5.4)$ & $<0.001$ \\
\hline Gynoid fat mass (\%) & $37.5[15.6]$ & $50.6[8.7]$ & $<0.001$ \\
\hline SBP $(m m H g)$ & $124.0(16.1)$ & $139.2(19.3)$ & $<0.001$ \\
\hline $\mathrm{DBP}(\mathrm{mmHg})$ & $71.1(11.0)$ & $78.0(10.9)$ & 0.001 \\
\hline Glucose (mg/dL) & $95.0[13.0]$ & $96.0[13.5]$ & 0.284 \\
\hline $\mathrm{HbA1c}(\%)$ & $5.5(0.3)$ & $5.6(0.3)$ & 0.016 \\
\hline Cholesterol (mg/dL) & $200.0[48.0]$ & $183.5[57.0]$ & 0.043 \\
\hline LDL-cholesterol (mg/dL) & $119.0[48.0]$ & $112.0[58.8]$ & 0.634 \\
\hline HDL-cholesterol (mg/dL) & $63.9(17.5)$ & $53.5(12.0)$ & 0.005 \\
\hline Triglycerides (mg/dL) & $79.0[39.0]$ & $111.5[75.0]$ & $<0.001$ \\
\hline hsCRP (mg/dL) & $0.7[0.9]$ & $4.9[7.2]$ & $<0.001$ \\
\hline K03695 (AU) & $1.6 \times 10^{-1}\left(3.0 \times 10^{-2}\right)$ & $1.4 \times 10^{-1}\left(2.4 \times 10^{-2}\right)$ & $8.0 \times 10^{-6}$ \\
\hline
\end{tabular}

\section{Gut bacterial ClpB-like gene function and microbiota composition and metabolites}

Gut bacterial ClpB-like gene function was found to be associated with bacterial metabolites in feces and plasma samples. To note, $\mathrm{ClpB}$ was positively associated with hippuric acid $\left(r=0.398, p=2.85 \times 10^{-4}\right)$ and 3indolepropionic acid $(r=0.259, p=0.034)$ in plasma and lupeol in feces $(r=0.242, p=0.039)$ and negatively with cholic acid in feces $(r=-0.317, p=0.008)$ (Fig. 2a, b) and a common gut microbiota ecosystem was simultaneously associated with these metabolites and ClpBlike gene function (Fig. 2c).

The bacterial families with the highest number of reads that contributed most to the ClpB-like gene function were the following: Clostridiaceae, Ruminococaceae, Lachnospiraceae, uncultured and not assigned Firmicutes, and uncultured Clostridiales within the Firmicutes phylum and Prevotellaceae, Bacteroidaceae, and Rikenellaceae within Bacteroidetes phylum (Fig. 3a; Additional file 1). Interestingly, while the relative abundance (RA) of not assigned Firmicutes (Fig. 3b), Rikenellaceae (Fig. 3c), and Clostridiaceae (Fig. 3d) families were lower in subjects with obesity, these families were also positively associated with gut bacterial ClpB-like gene function (not assigned Firmicutes $\left(r=0.405\right.$, FDR $=2.93 \times 10^{-2}$ ), Rikenellaceae $(r=0.217, \mathrm{FDR}=0.031)$, and
Clostridiaceae $(r=0.239, \mathrm{FDR}=0.017))$. Importantly, the associations between ClpB-like gene function and some families Clostridiaceae $\left(r=0.352, p=4.2 \times 10^{-5}\right)$, Ruminococaceae $(r=0.284, p=0.001)$, not assigned Firmicutes $\left(r=0.523, p=2.1 \times 10^{-10}\right)$, and uncultured Firmicutes $\left(r=0.367, p=1.8 \times 10^{-5}\right)$ remained significant even after adjusting for body mass index.

Focusing on the $\alpha-\mathrm{MSH}$ molecular mimicry, the families that most contributed to the ClpB-like gene function (Fig. 3a) and were also detected in relative lower abundance in subjects with obesity (Fig. 3b-d), such as not assigned Firmicutes, Rikenellaceae, and Clostridiaceae showed 48,192 and 175 sequences with 100\% homology with $\alpha-\mathrm{MSH}$ epitope of $E$. coli with a total mean of identity of $82.13 \%, 92.25 \%$, and $81.36 \%$, respectively (Additional files 2-3). Other families, that also have an important contribution to ClpB-like gene function, such as uncultured Clostridiales, Ruminococcaceae, Lachnospiraceae, uncultured Firmicutes, Prevotellaceae, uncultured Bacteria, and Bacteroidaceae (Fig. 3a) presented 1068, 766, 855, 265, 262, 149, and 431 sequences with a total homology with motif in E. coli and a total mean of identity of $89.04 \%, 85.04 \%, 88.28 \%, 86.44 \%$, $95.78 \%, 90.01 \%$, and $93.64 \%$, respectively (Additional files 2-3). 

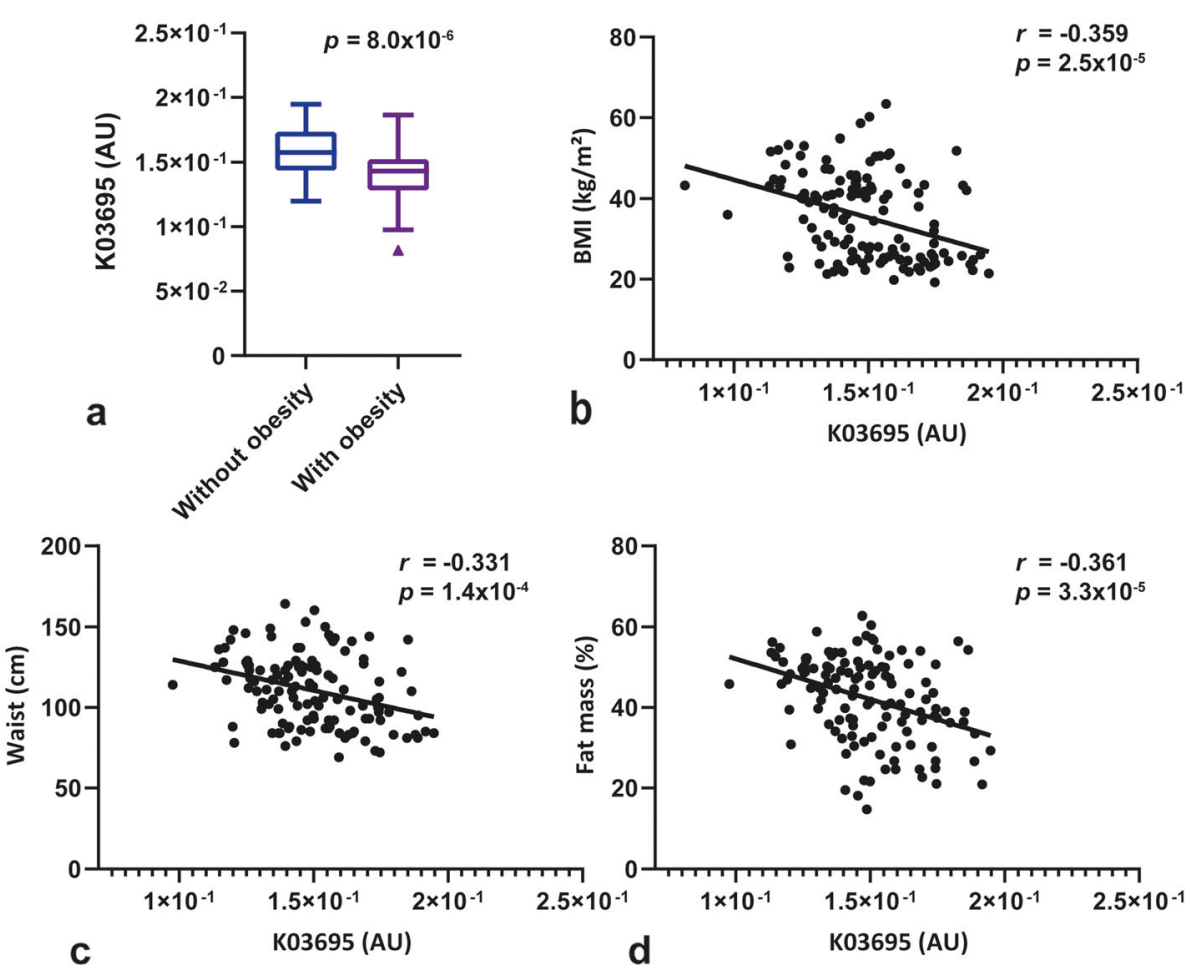

Fig. 1 Gut bacterial ClpB-like gene function is associated with decreased body weight in humans. a Box plot showing the differences of gut bacterial ClpB-like gene function (K03695, KEGG annotation) between subjects without (body mass index, BMI 18.5-30 kg/m²) and with obesity $\left(\mathrm{BMI} \geq 30 \mathrm{~kg} / \mathrm{m}^{2}\right.$ ). b Scatterplot displaying the relationship between BMI and gut bacterial ClpB-like gene function. c Scatterplot of the relationship between waist circumference and gut bacterial ClpB-like gene function. $\mathbf{d}$ Scatterplot of the relationship between the percentage of total fat mass assessed by DEXA and gut bacterial ClpB-like gene function

Furthermore, the bacterial families whose reads more contributed to the ClpB-like gene function were also associated with some bacterial metabolites (Fig. 2c). Remarkably, a cluster of metabolites and not assigned Firmicutes, Rikenellaceae, and Clostridiaceae, among others, were identified (Fig. 2c).

\section{Gut microbiota transplantation in mice}

Finally, we tested the gut bacterial ClpB-like gene function in 33 mice with no significant differences in initial body weight (Fig. 3e). After antibiotic treatment, 11 mice were orally gavaged with vehicle and 22 with fecal material from 22 subjects paired in age and sex. ClpB-like gene function in mice showed a trend to be negatively associated with body weight gain as the time of the experiment passed (Fig. 3f) and also a negative tendency to be associated with percent weight gain at the end of the experiment (Fig. 3g). ClpB-like gene function in humans was not significantly associated with ClpB-like gene function in mice. Nevertheless, the families that more contributed to ClpB-like gene function in humans were also associated with ClpB-like gene function in mice after adjusting for the donor's body mass index, such as not assigned Firmicutes $(r=0.621, p=0.003)$, uncultured Firmicutes $(r=0.529, p=0.014)$, Prevotellaceae $\left(r=0.725, p=4.1 \times 10^{-7}\right)$, Rikenellaceae $(r=0.702$, $\left.p=3.9 \times 10^{-4}\right)$, and Ruminococcaceae $(r=0.526, p=$ 0.014). Furthermore, two of the bacterial families that more contributed to the gut bacterial ClpB-like function in humans, Clostridiaceae $(r=-0.445, p=0.038)$ and Prevotellaceae ( $r=-0.479, p=0.024$ ) were negatively associated with weight gain in mice (Fig. $3 \mathrm{~h}$ ). The absolute abundance (AA) of Prevotellaceae in mice was also positively associated with the gut bacterial ClpB-like gene function in mice (Fig. 3i). A deeper analysis using DESeq2 [13] identified species of Prevotellaceae, both negatively associated with mice' weight in the 7th week of follow-up (Fig. 3j) and positively with gut bacterial ClpB-like gene function (Fig. 3k).

\section{Discussion}

Gut bacterial ClpB-like gene function in feces (KEGG annotation: K03695) was associated with decreased body weight, central obesity, and total fat mass in humans. Accordingly, this gut bacterial function was found in lower relative abundance in subjects with obesity. These results are in line with previous evidence in mouse models and in sillico findings. In a recent study, the 


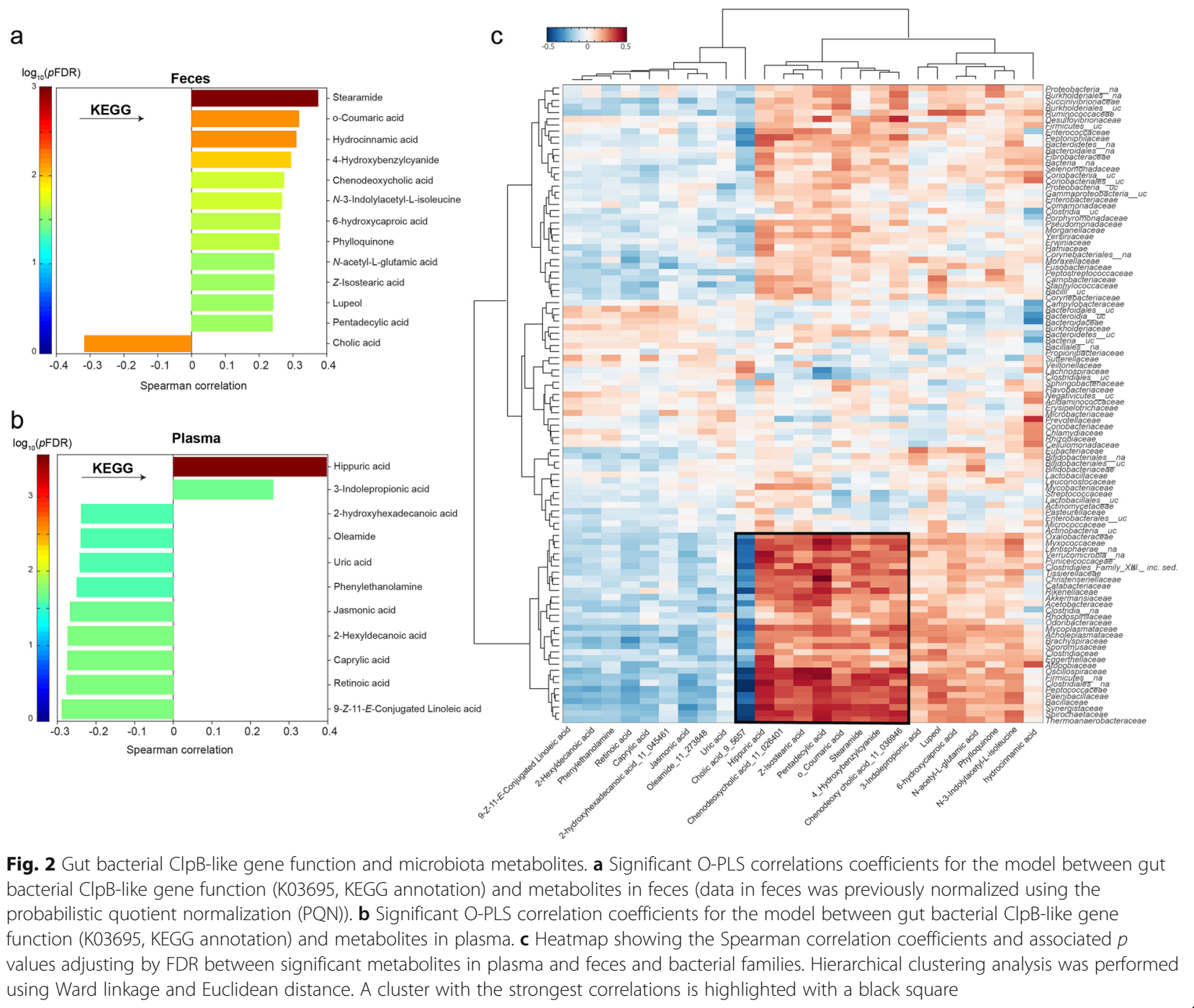

administration of a probiotic, Hafnia alvei, a ClpBproducing bacterium reduced fat mass and food intake in $o b / o b$ and after a high-fat diet in mice [12] and also improved their metabolic profile [14]. In this study, the enterobacterial $\mathrm{ClpB}$ gene was also tested in sillico analysis of fecal metagenomes from the MetaHIT database [12]. To our knowledge, our study sets up the first evidence of the association of ClpB gene function and obesity in a clinical setting.

The caseinolytic protease $\mathrm{B}(\mathrm{ClpB})$ protein described in $E$. coli is an ATP-dependent ring forming chaperone that mediates the resolubilization of aggregated proteins [15]. This gut bacteria $\mathrm{ClpB}$ protein has been identified as a conformational antigen-mimetic of $\alpha-\mathrm{MSH}$ that could activate the MC4R in the hypothalamus ultimately promoting the anorexigenic pathway [10]. In that sense, autoantibodies that interreacted with the $\alpha-\mathrm{MSH}$ were found elevated in subjects with anorexia and bulimia nervosa [16, 17], and plasma levels of $\mathrm{ClpB}$ were proportional to ClpB DNA in feces [9]. In our study, metagenomic analysis of fecal microbiota revealed that the RA of Rikenellaceae, Clostridiaceae and not assigned Firmicutes were positively associated with $\mathrm{ClpB}$ gene function and were detected in lower relative abundance in subjects with obesity. Tennoune et al. firstly demonstrated the $\alpha-\mathrm{MSH}$ mimetic $\mathrm{ClpB}$ protein in E. coli from the phylum Proteobacteria [10]. Other sources of $\alpha$ MSH-like epitope of E.coli ClpB identified in sillico analysis were bacteria belonging to the genera Escherichia, Salmonella, Shigella, Klebsiella, Enterobacter, Citrobacter, Cronobacter, and Hafnia, and in that search, the ClpB appeared to be specific to the family Enterobacteriaceae and in some Brassicaceae [18]. However, except for E. coli $\mathrm{ClpB}$, we did not find studies that have tested the properties of this protein in the previous genera described. Other bacteria that present sequence homology in at least five amino acids with $\alpha-\mathrm{MSH}$ have been reported, such as bacteria belonging to the phyla 


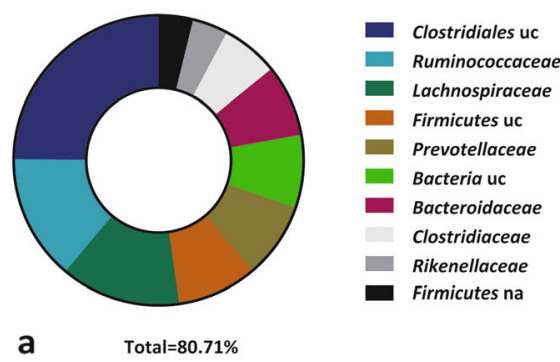

a

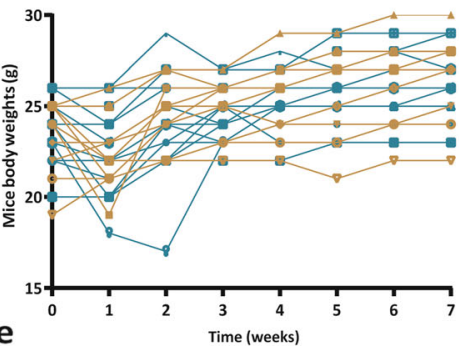

$-M_{1 c}+M_{12 t} \rightarrow m_{23}$ $=\mathrm{m}_{20} \rightarrow \mathrm{w}_{13 \mathrm{t}} \rightarrow \mathrm{m}_{24 \mathrm{t}}$ - M3c * M14t $+\mathrm{M}_{25 \mathrm{t}}$ - Mac $-M 15 t-M 26 t$ - M5C - M16t - M271 - Mec $-M 17 t-m 28$ M6c - M17t - M221 - Mrc - Mrat a maso Mbc M - M19t - M30 M9c \& M2Ot - M31 M10c \& M21t \& M321 $-\mathrm{M} 11 \mathrm{c}-\mathrm{M} 22 \mathrm{t}-\mathrm{M} 3 \mathrm{I}$

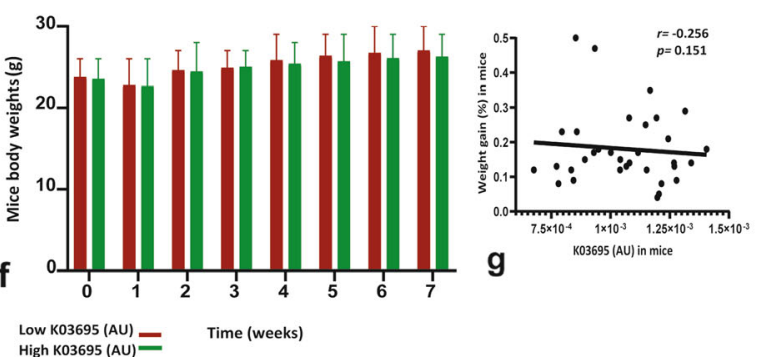

$p=1.4 \times 10^{-5}$
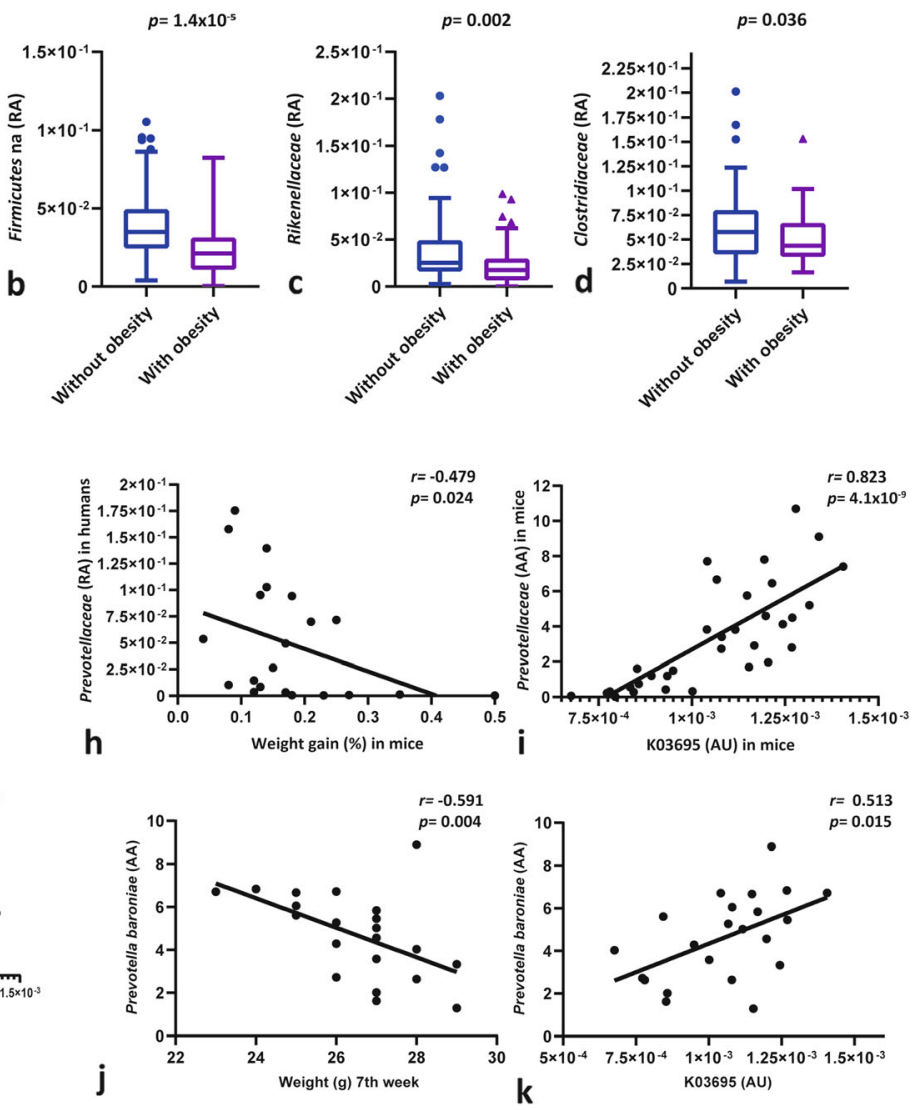

Fig. 3 Gut bacterial ClpB-like gene function and metagenomics. a Top bacterial taxa at the family level contributing reads to the gut bacterial ClpB-like gene function, in total relative figures per sample (uncultured, uc; not assigned, na). b-d Box plots showing the differences of some of the top ten bacterial families (relative abundance, RA) contributing to the gut bacterial ClpB-like gene function in subjects with and without obesity. e Mice body weights after gut microbiota transplantation. Controls (mouse, M; control, c) are shown in yellow ( $n=11$ ) and mice which received microbiota from human donors (transplant, t) are shown in blue $(n=22)$. $\mathbf{f}$ Bar chart showing mean and standard deviation mice body weights, regarding the median of the gut bacterial ClpB-like gene function detected in mice, K03695 (arbitrary units, AU). g Scatterplot showing the relationship between percent weight gain in mice (calculated as (body weight final (g) —body weight initial (g))/body weight initial (g)*100) and gut bacterial ClpB-like gene function, K03695 (AU), in mice. $\mathbf{h}$ Scatterplot showing the relationship between percent weight gain in mice and Prevotellaceae RA in humans. i Scatterplot showing the relationship between gut bacterial ClpB-like gene function (K03695, KEGG annotation) and Prevotellaceae absolute abundance (AA) in mice. $\mathbf{j}$ Scatterplot showing the relationship between the final weight (7th week) in mice and Prevotellaceae baroniae AA in humans. k Scatterplot showing the relationship between gut bacterial ClpB-like gene function (K03695, KEGG annotation) in mice and Prevotellaceae baroniae AA in human donors

Bacteroidetes, Actinobacteria (Bifidobacterium longum, Frankia), Firmicutes (Bacillus cereus, Clostridium tetani), or Proteobacteria (Helicobacter) [19]. In our study, we have identified that the sequences of the bacterial families which more contributed to gut bacterial ClpB-like gene function presented the motif of $\alpha-\mathrm{MSH}-$ like epitope of E.coli ClpB, suggesting that this mechanism could be shared with other bacterial families and might be explain the role on body weight regulation. However, the presence of amino-acid sequence homology does not imply the same molecular function and experimental studies are needed to test the $\alpha-\mathrm{MSH}$ mimicry of the different $\mathrm{ClpB}$ proteins from other microbial organisms [18]. Nonetheless, there is some evidence that gut bacterial ClpB-like gene function, either by the same $\alpha$-MSH-like epitope of ClpB E.coli with an effect on the anorexigenic pathway [18] or by others ClpB fragments with other unknown routes implicated, seems to have a role in the pathophysiology of obesity attributed to the gut microbiota.

In addition, we found a relationship between the diet and the detection of ClpB-like gene function in humans. In general, a negative association was found between total energy intake and gut bacterial ClpB-like gene function. This data is concordant with previous evidence in mice in which the dietary restriction induced an increase of ClpB [20]. On the contrary, we observed positive associations between ClpB-like gene function and different macronutrients and fiber. Proteins have been identified as the main contributors to the ClpB E.coli 
levels in in vitro studies [21]. In our study, positive relationships were found not only with total protein intake but also with fiber intake and the rest of macronutrients. However, the strength of the body mass index appeared to be more important than the role of each macronutrient separately. Interestingly, ClpB-like gene function was associated with some metabolites in plasma such as hippuric acid and 3-indolepropionic acid, or lupeol and cholic acid in feces. The direction of the relationships is in line with current evidence. Higher levels of hippuric acid have been associated with a beneficial metabolic profile, decreased fasting plasma glucose and insulin secretion [22]. Similar observations have been made regarding 3-indolepropionic acid [23]. Lupeol has exhibited antioxidant, anti-inflammatory, anti-hyperglycemic, hypolipemiant, and anti-mutagenic properties [24].

In the animal experiment, microbial families that seem to contribute to the gut bacterial ClpB-like gene function, such as Prevotellaceae and Clostridiaceae were negatively associated with weight gain during the 7 weeks of follow-up. Despite not directly observing a negative significant association between gut bacterial ClpB-like gene function and weight gain in mice, it can be seen a tendency as the time of the experiment passed. Maybe, if the duration of the study would have been longer or the mice sample size larger, a significant association would be reached. In any case, the direction of the association between gut bacterial ClpB-like gene function and body weight seems to be shared between humans and mice. This would be the first study investigating the effects of microbiota transplantation from humans to mice evaluating $\mathrm{ClpB}$ function. Previous reports in mice were based on chronic intragastric delivery of E.coli [10] and H. alvei [12].

\section{Conclusions}

$\mathrm{ClpB}$ protein has been previously studied in animal models $[9,10]$. Antibody anti-ClpB was investigated in a single human study [10]. This is the first study, to our knowledge, evaluating gut bacterial ClpB-like gene function in human subjects linked to obesity status, gut bacterial ecosystem, and diet. The findings of the current study show that a bacterial ecosystem enriched in ClpB-like gene function that was negatively associated with body mass index, waist circumference, and fat mass, and detected in lower abundance in subjects with obesity. We speculate that this gut bacterial ClpB-like function could lead to increased satiety and decreased fat mass in the long term.

\section{Methods}

\section{Clinical study}

Recruitment of study subjects

From January 2016 to October 2017, a cross-sectional case-control study was undertaken in the Endocrinology
Department of Josep Trueta University Hospital. We included consecutive subjects with obesity (body mass index, BMI $\geq 30 \mathrm{~kg} / \mathrm{m}^{2}$ ) and age- and sex-matched nonobese subjects (BMI $18.5-30 \mathrm{~kg} / \mathrm{m}^{2}$ ), with an age range of 27.2-66.6 years. Exclusion criteria were: type 2 diabetes mellitus, chronic inflammatory systemic diseases, acute, or chronic infections in the previous month; use of antibiotic, antifungal, antiviral, or treatment with proton-pump inhibitors; severe disorders of eating behavior or major psychiatric antecedents; or excessive alcohol intake $(\geq 40 \mathrm{gOH} /$ day in women or $80 \mathrm{gOH} /$ day in men). The institutional review board-Ethics Committee and the Committee for Clinical Research (CEIC) of Dr. Josep Trueta University Hospital (Girona, Spain)-approved the study protocol and informed written consent was obtained from all participants.

\section{Clinical and laboratory parameters}

Body composition was assessed using a dual energy x-ray absorptiometry (DEXA, GE lunar, Madison, Wisconsin).

\section{Dietary pattern}

The dietary characteristics of the subjects were collected in a personal interview using a validated food-frequency questionnaire [25].

\section{Extraction of fecal genomic DNA and whole-genome shotgun sequencing}

Total DNA was extracted from frozen human stools using the QIAamp DNA mini stool kit (Qiagen, Courtaboeuf, France). Quantification of DNA was performed with a Qubit 3.0 fluorometer (Thermo Fisher Scientific, Carlsbad, CA, USA), and $1 \mathrm{ng}$ of each sample $(0.2 \mathrm{ng} / \mu \mathrm{L})$ was used for shot gun library preparation for highthroughput sequencing, using the Nextera DNA Flex Library Prep kit (Illumina, Inc., San Diego, CA, USA) according to the manufacturers' protocol. Sequencing was carried out on a NextSeq 500 sequencing system (Illumina) with $2 \times 150$-bp paired-end chemistry, at the facilities of the Sequencing and Bioinformatic Service of the FISABIO (Valencia, Spain).

The obtained input fastq files were decompressed, filtered, and 3 ends-trimmed by quality, using prinseq-lite0.20.4 program [26] and overlapping pairs were joined using FLASH-1.2.11 [27]. Fastq files were then converted into fast files, and human and mouse host reads were removed by mapping the reads against the GRCh38.p11, reference human genome (Dec 2013), and GRCm38.p6, reference mouse genome (Sept 2017), respectively, by using bowtie2-2.3.4.3 [28] with end-to-end and very sensitive options.

Next, functional analyses were carried out by assembling the non-host reads into contigs by MEGAHIT 
v1.1.2 [29] and mapping those reads against the contigs with bowtie 2 . Reads that did not assemble were appended to the contigs. Next, the program Prodigal v2.6.342 [30] was used for predicting codifying regions. Functional annotation was carried out with HMMER [31] against the Kyoto Encyclopedia of Genes and Genomes (KEGG) database, version 2016 [32] to obtain the functional subcategory, route, and annotation of the genes. An in-house pipeline, consisting of a series of consecutive customized scripts, implemented by the statistical package $\mathrm{R}$ 3.1.0 [33], coordinated and concatenated by shell commands, was applied to the functionally annotated reads to first parse the HMMER table, and next to get the best annotation per open reading frame (ORF) and to identify which ORFs were annotated, followed by getting the nucleotides and amino acids sequences. Next, it allowed us to get non-singleton contigs with annotated ORFs (annotated contigs), add the annotation for each ORF, get the reads inside that annotated ORFs, count the alignments per annotation, count alignment per category (a specific annotation can belong to multiple categories), and finally build a contingency table to which descriptions were added.

Taxonomic annotation was implemented with Kaiju v1.6.2 [34] on the human and mouse-free reads. Addition of lineage information was added, counting of taxa and generation of an abundance matrix for all samples were performed using the package $\mathrm{R}$.

\section{Molecular mimicry}

The mimetic amino acid motif of the $\alpha$-MSH peptide (RWGKPV) was first aligned to the ClpB from E. coli str K12 (accession number NP_417083.1). Then, the predicted ORFs from each sequence were aligned with Clustal Omega [35] to the previous two sequences. Those that encompassed the region that contained the epitope-like sequence were filtered, discarding reads which not included it. Sequences beyond 30 amino acids before and after the aligned $\alpha-\mathrm{MSH}$ peptide were trimmed and 40 alignments were plotted. R scripts [33] allowed us to create a table with the aligned motif and calculate the identity between the E. coli and the microbial counterparts in the sequences, as well as, assign the taxonomy, at family level, to each contig or single read. The average identity of the epitope in each bacterial family compared to the motif in E. coli as well as the distribution of families through the sequences according to their degree of homology were also calculated with $R$ package [33].

\section{Metabolomics analyses}

For non-targeted metabolomics analysis, metabolites were extracted from fecal samples with methanol according to previously described methods [36], by using
phenylalanine-C13 as internal standard. After $1 \mathrm{~h}$ at $20^{\circ} \mathrm{C}$, methanol supernatants were centrifuged at $12000 \mathrm{~g}$ for $3 \mathrm{~min}$ and evaporated using a Speed Vac (Thermo Fisher Scientific, Barcelona, Spain). The resulting pellets were resuspended in water with $0.4 \%$ acetic acid/methanol $(50 / 50)$.

We used an ultra-high-pressure liquid chromatography (UHPLC) with an Agilent 1290 LC system coupled to an electrospray-ionization quadruple time of flight mass spectrometer (Q-TOF) 6520 instrument (Agilent Technologies, Barcelona, Spain). A column with $1.8 \mu \mathrm{m}$ particle size was employed and we performed the preliminary identification of differential metabolites by using the database PCDL from Agilent (Agilent Technologies, Barcelona, Spain), which uses retention times, exact mass, and isotope distribution in a standardized chromatographic system as an orthogonal searchable parameter to complement accurate mass data (AMRT approach) according to previously published works [37].

\section{Animal procedures}

Male C57BL/6 J mice (Charles River, France), weighing 29-26 g at the beginning of the experiment were used in this study. Mice were housed individually in controlled laboratory conditions with the temperature maintained at $21 \pm 1{ }^{\circ} \mathrm{C}$ and humidity at $55 \pm 10 \%$ during all the study. Animal procedures were conducted in strict accordance with the guidelines of the European Communities Directive 86/609/EEC regulating animal research and were approved by the local ethical committee (CEEA-PRBB). All the experiments were performed under blinded conditions.

Then, mice were given a cocktail of ampicillin and metronidazole, vancomycin (all at $500 \mathrm{mg} / \mathrm{L}$ ), ciprofloxacin $\mathrm{HCl}(200 \mathrm{mg} / \mathrm{L})$, and imipenem $(250 \mathrm{mg} / \mathrm{L})$ once daily for 14 consecutive days in drinking water, as previously described [38]. Seventy-two hours later, animals were colonized via daily oral gavage of donor microbiota $(150 \mu \mathrm{L})$ for 3 days. Animals were orally gavaged with fecal material from healthy volunteers $(n=22)$. To offset potential confounder and/or cage effects and to reinforce the donor microbiota phenotype, booster inoculations were given twice per week throughout the study. Animals were maintained on normal mouse chow diet (Rat and Mouse No. 1 Maintenance Diet, Special Diet Services, Essex, UK) and water ad libitum, and were weighted every week during 7 weeks. Food intake was the same in the two groups. At the end of the study, the animals were consecutively sacrificed. The cecum was removed, weighted, and stored, and the feces collected and stored at $-80^{\circ} \mathrm{C}$ for microbiota analysis. 


\section{Statistical analysis}

Firstly, normal distribution and homogeneity of variances were tested. Results are expressed as number and frequencies for categorical variables, mean and standard deviation (SD) for normal distributed continuous variables, and median and interquartile range [IQR] for nonnormal distributed continuous variables. To determine differences between study groups, we used $x^{2}$ for categorical variables, unpaired Student's $t$ test in normal quantitative, and Mann-Whitney $U$ test for non-normal quantitative variables. Nonparametric Spearman analysis was used to determine the correlation between quantitative variables. These analyses were performed using SPSS version 24 (IBM Corp., Armonk, NY) and GraphPad Prism version 8.00 (GraphPad Software, La Jolla, CA, USA).

Metabolomics data were analyzed using in-house MATLAB scripts. Orthogonal projection to latent structures (O-PLS) using unit variance scaling was then applied to identify metabolites associated with the gut bacterial ClpB-like gene function. Metabolites were used as the descriptor matrices $(\mathrm{X})$ and gut bacterial $\mathrm{ClpB}$ like gene function was used as the response $(\mathrm{Y})$. The predictive performance $\left(Q^{2} \mathrm{Y}\right)$ of each model was calculated using a seven-fold cross-validation approach and model validity was established by permutation testing (1000 permutations). Significance of O-PLS correlation coefficients was adjusted by the Benjamini-Hochberg method (FDR) [39].

Bacterial taxa associated with the gut bacterial ClpBlike gene function in humans were identified using the DESeq2 $R$ packages [13]. Taxa were previously filtered so that only those with more than 10 reads in at least two samples were selected.

\section{Supplementary information}

Supplementary information accompanies this paper at https://doi.org/10. 1186/s40168-020-00837-6.

Additional file 1. Top 10 bacterial taxa (family level) contributing reads to the gut bacterial ClpB-like function, in absolute and relative figures per sample.

Additional file 2. Alignments of the mimetic amino acid motif of the aMSH, the ClpB from E. coli str K12 and the predicted ORFs from each sequence of the samples 1, 30 and 143.

Additional file 3. Alignments. Alignment motif of the a-MSH and percentage of identity between the E. coli motif and the identified microbial counterparts in each sequence. Bacterial family identity: Average identity of the epitope in each bacterial family compared to the motif in E. coli as well as the distribution of families through the samples according to their degree of homology.

\section{Abbreviations}

a-MSH: a-melanocyte-stimulating hormone; AA: Absolute abundance; AgRP: Agouti-related protein; BMl: Body mass index; ClpB: Caseinolytic peptidase B protein homolog; GLP-1: Glucagon-like peptide 1; MC4R: Melanocortin-4 receptor; NPY: Neuropeptide Y;

POMC: Proopiomelanocortin; PYY: Peptide YY; RA: Relative abundance

\section{Acknowledgements}

We are in debt with the subjects involved in this project. We also thank Emili Loshuertos and Oscar Rovira for their help in the recruitment of the subjects. We want to particularly acknowledge the patients, the FATBANK platform promoted by the CIBEROBN, and the IDIBGI Biobank (Biobanc IDIBGI, B.0000872), integrated in the Spanish National Biobanks Network, for their collaboration and coordination.

\section{Authors' contributions}

M.A-R. researched the data, performed the statistical analysis, and wrote the manuscript. J.M-P. performed part of the statistical analysis. V.P-B and A.M. determined and analyzed the microbiota and contributed to write the corresponding parts of the manuscript associated with these data. A.B. and R.M. performed the experiments in mice and contributed to write the corresponding parts of the paper that are associated with the mice data. M.P-O performed the metabolomics analysis. W.R. contributed to the discussion and reviewed the manuscript. J.M.F.-R. carried out the conception and coordination of the study, performed the statistical analysis, and wrote the manuscript. All authors read and approved the final manuscript. J.M.F.-R. is the guarantor of this work and, as such, had full access to all the data in the study and takes responsibility for the integrity of the data and the accuracy of the data analysis.

\section{Funding}

This work was partially supported by research grants FIS (PI15/01934) from the Instituto de Salud Carlos III from Spain, SAF2015-65878-R from Ministry of Economy and Competitiveness, Prometeo/2018/A/133 from Generalitat Valenciana, Spain and also by Fondo Europeo de Desarrollo Regional (FEDER) funds, European Commission (FP7, NeuroPain \#2013-602891), the Catalan Government (AGAUR, \#SGR2017-669, ICREA Academia Award 2015), the Instituto de Salud Carlos III (RTA, \#RD16/0017/0020), and the European Regional Development Fund (No. 01.2.2-LMT-K-718-02-0014). María ArnoriagaRodríguez is funded by Instituto de Salud Carlos III, Río Hortega (CP19/ 00190). Jordi Mayneris-Perxachs is funded by Instituto de Salud Carlos III, Miguel Servet (CP18/00009). The project has also been 65\% cofinanced by the European Regional Development Fund (ERDF) through the Interreg V-A Spain-France-Andorra programme (POCTEFA 2014-2020). POCTEFA aims to reinforce the economic and social integration of the French-Spanish-Andorran border. Its support is focused on developing economic, social and environmental cross-border activities through joint strategies favouring sustainable territorial development.

\section{Availability of data and materials}

The data that support the findings of this study are available from the corresponding author upon reasonable request.

\section{Ethics approval and consent to participate}

Human study: The institutional review board-Ethics Committee and the Committee for Clinical Research (CEIC) of Dr. Josep Trueta University Hospital (Girona, Spain) -approved the study protocol and informed written consent was obtained from all participants.

Mice experiment: Animal procedures were conducted in strict accordance with the guidelines of the European Communities Directive 86/609/EEC regulating animal research and were approved by the local ethical committee (CEEA-PRBB).

\section{Consent for publication}

Not applicable.

\section{Competing interests}

The authors declare that they have no competing interests.

\section{Author details}

'Department of Diabetes, Endocrinology and Nutrition, Dr. Josep Trueta University Hospital, Carretera de França s/n, 17007 Girona, Spain. ${ }^{2}$ Nutrition, Eumetabolism and Health Group, Girona Biomedical Research Institute (IdibGi), Girona, Spain. ${ }^{3}$ CIBER Fisiopatología de la Obesidad y Nutrición (CIBEROBN), Madrid, Spain. ${ }^{4}$ Department of Medical Sciences, Faculty of Medicine, University of Girona, Girona, Spain. ${ }^{5}$ Laboratory of Neuropharmacology, Department of Experimental and Health Sciences, Universitat Pompeu Fabra, Barcelona, Spain. ${ }^{6}$ Present address: Institute of 
Biochemistry, Life Sciences Center, Vilnius University, Saulètekio av. 7, LT-10257 Vilnius, Lithuania. ${ }^{7}$ Department of Genomics and Health, Foundation for the Promotion of Health and Biomedical Research of Valencia Region (FISABIO-Public Health), Valencia, Spain. ${ }^{8}$ Biomedical Research Networking Center for Epidemiology and Public Health (CIBERESP), Madrid, Spain. ${ }^{9}$ Institute for Integrative Systems Biology (I2SysBio), University of Valencia, Spanish National Research Council (CSIC-UVEG), Valencia, Spain. ${ }^{10}$ Metabolic Pathophysiology Research Group, Lleida Biomedical Research Institute (IRBLleida), Universitat de Lleida, Lleida, Spain. ${ }^{11}$ Hospital del Mar Medical Research Institute (IMIM), Barcelona, Spain.

\section{Received: 6 February 2020 Accepted: 2 April 2020}

\section{Published online: 30 April 2020}

\section{References}

1. Maruvada P, Leone V, Kaplan LM, Chang EB. The human microbiome and obesity: moving beyond associations. Cell Host Microbe. 2017;22:589-99.

2. Bouter KE, van Raalte DH, Groen AK, Nieuwdorp M. Role of the gut microbiome in the pathogenesis of obesity and obesity-related metabolic dysfunction. Gastroenterology. 2017;152:1671-8.

3. Torres-Fuentes C, Schellekens H, Dinan TG, Cryan JF. The microbiota-gutbrain axis in obesity. Lancet Gastroenterol Hepatol. 2017;2:747-56.

4. Burokas A, Moloney RD, Dinan TG, Cryan JF. Microbiota regulation of the mammalian gut-brain axis. Adv Appl Microbiol. 2015;91:1-62.

5. Gribble FM, Reimann F. Function and mechanisms of enteroendocrine cells and gut hormones in metabolism. Nat Rev Endocrinol. 2019;15:226-37.

6. Cowley MA, Pronchuk N, Fan W, Dinulescu DM, Colmers WF, Cone RD. Integration of NPY, AGRP, and melanocortin signals in the hypothalamic paraventricular nucleus: evidence of a cellular basis for the adipostat. Neuron. 1999;24:155-63.

7. Cani PD, Van Hul M, Lefort C, Depommier C, Rastelli M, Everard A. Microbial regulation of organismal energy homeostasis. Nat Metab. 2019;1:34-46.

8. Turnbaugh PJ, Ley RE, Mahowald MA, Magrini V, Mardis ER, Gordon JI. An obesity-associated gut microbiome with increased capacity for energy harvest. Nature. 2006;444:1027-31.

9. Breton J, Tennoune N, Lucas N, Francois M, Legrand R, Jacquemot J, et al. Gut commensal E. coli proteins activate host satiety pathways following nutrient-induced bacterial growth. Cell Metab. 2016;23:324-34.

10. Tennoune N, Chan P, Breton J, Legrand R, Chabane YN, Akkermann K, et al. Bacterial ClpB heat-shock protein, an antigen-mimetic of the anorexigenic peptide a-MSH, at the origin of eating disorders. Transl Psychiatry. 2014;4: e458.

11. Kühnen $\mathrm{P}$, Krude $\mathrm{H}$, Biebermann $\mathrm{H}$. Melanocortin-4 receptor signalling: importance for weight regulation and obesity treatment. Trends Mol Med. 2019;25:136-48.

12. Legrand R, Lucas N, Dominique M, Azhar S, Deroissart C, Le Solliec M-A, et al. Commensal Hafnia alvei strain reduces food intake and fat mass in obese mice-a new potential probiotic for appetite and body weight management. Int J Obes. 2020. https://doi.org/10.1038/s41366-019-0515-9.

13. Love Ml, Huber W, Anders S. Moderated estimation of fold change and dispersion for RNA-seq data with DESeq2. Genome Biol. 2014;15:550.

14. Lucas N, Legrand R, Deroissart C, Dominique M, Azhar S, Le Solliec M-A, et al. Hafnia alvei HA4597 strain reduces food intake and body weight gain and improves body composition, glucose, and lipid metabolism in a mouse model of hyperphagic obesity. Microorganisms. 2019. https://doi.org/10. 3390/microorganisms8010035.

15. Mogk A, Schlieker C, Strub C, Rist W, Weibezahn J, Bukau B. Roles of individual domains and conserved motifs of the AAA+ chaperone ClpB in oligomerization, ATP hydrolysis, and chaperone activity. J Biol Chem. 2003; 278:17615-24

16. Breton J, Legrand R, Akkermann $K$, Järv A, Harro J, Déchelotte $P$, et al. Elevated plasma concentrations of bacterial ClpB protein in patients with eating disorders. Int J Eat Disord. 2016;49:805-8.

17. Fetissov SO, Harro J, Jaanisk M, Järv A, Podar I, Allik J, et al. Autoantibodies against neuropeptides are associated with psychological traits in eating disorders. Proc Natl Acad Sci U S A. 2005;102:14865-70.

18. Fetissov SO, Legrand R, Lucas N. Bacterial protein mimetic of peptide hormone as a new class of protein- based drugs. Curr Med Chem. 2019;26: 546-53.

19. Fetissov SO, Hamze Sinno M, Coëffier M, Bole-Feysot C, Ducrotté P, Hökfelt $T$, et al. Autoantibodies against appetite-regulating peptide hormones and neuropeptides: putative modulation by gut microflora. Nutrition. 2008;24: 348-59.

20. Dominique M, Legrand R, Galmiche M, Azhar S, Deroissart C, Guérin C, et al. Changes in microbiota and bacterial protein caseinolytic peptidase $b$ during food restriction in mice: relevance for the onset and perpetuation of anorexia nervosa. Nutrients. 2019;11:2514.

21. Dominique $M$, Breton J, Guérin C, Bole-Feysot C, Lambert G, Déchelotte $P$, et al. Effects of macronutrients on the in vitro production of $\mathrm{ClpB}, \mathrm{a}$ bacterial mimetic protein of a-MSH and its possible role in satiety signaling. Nutrients. 2019;11:2115.

22. de Mello VDF, Lankinen MA, Lindström J, Puupponen-Pimiä R, Laaksonen DE, Pihlajamäki J, et al. Fasting serum hippuric acid is elevated after bilberry (Vaccinium myrtillus) consumption and associates with improvement of fasting glucose levels and insulin secretion in persons at high risk of developing type 2 diabetes. Mol Nutr Food Res. 2017. https://doi.org/10. 1002/mnfr.201700019.

23. Abildgaard A, Elfving B, Hokland M, Wegener G, Lund S. The microbial metabolite indole-3-propionic acid improves glucose metabolism in rats, but does not affect behaviour. Arch Physiol Biochem. 2018;124:306-12.

24. Tsai F-S, Lin L-W, Wu C-R. Lupeol and its role in chronic diseases. Adv Exp Med Biol. 2016;929:145-75.

25. Vioque J, Navarrete-Muñoz E-M, Gimenez-Monzó D, García-de-la-Hera M, Granado F, Young IS, et al. Reproducibility and validity of a food frequency questionnaire among pregnant women in a Mediterranean area. Nutr J. 2013;12:26.

26. Schmieder R, Edwards R. Quality control and preprocessing of metagenomic datasets. Bioinformatics. 2011;27:863-4.

27. Magoč T, Salzberg SL. FLASH: Fast length adjustment of short reads to improve genome assemblies. Bioinformatics. 2011;27:2957-63.

28. Langmead B, Salzberg SL. Fast gapped-read alignment with Bowtie 2. Nat Methods. 2012:9:357-9.

29. Li D, Liu CM, Luo R, Sadakane K, Lam TW. MEGAHIT: an ultra-fast singlenode solution for large and complex metagenomics assembly via succinct de Bruijn graph. Bioinformatics. 2015;31:1674-6.

30. Hyatt D, Chen GL, LoCascio PF, Land ML, Larimer FW, Hauser LJ. Prodigal: prokaryotic gene recognition and translation initiation site identification. BMC Bioinformatics. 2010;11:119.

31. Durbin R, Eddy SR, Krogh A, Mitchison G. Biological sequence analysis: probabilistic models of proteins and nucleic acids. Cambridge: Cambridge Univeristy Press; 1998.

32. Kanehisa M. KEGG: Kyoto Encyclopedia of Genes and Genomes. Nucleic Acids Res. 2000;28:27-30.

33. $\mathrm{R}$ Core Team. R: a language and environment for statistical computing. $\mathrm{R}$ Foundation for Statistical Computing. 2014. http://www.R-project.org.

34. Menzel P, Ng KL, Krogh A. Fast and sensitive taxonomic classification for metagenomics with Kaiju. Nat Commun. 2016;7:11257.

35. Madeira F, Park YM, Lee J, Buso N, Gur T, Madhusoodanan N, et al. The EMBL-EBI search and sequence analysis tools APIs in 2019. Nucleic Acids Res. 2019;47:W636-41.

36. Wikoff WR, Pendyala G, Siuzdak G, Fox HS. Metabolomic analysis of the cerebrospinal fluid reveals changes in phospholipase expression in the CNS of SIV-infected macaques. J Clin Invest. 2008;118:2661-9.

37. Sana TR, Roark JC, Li X, Waddell K, Fischer SM. Molecular formula and METLIN personal metabolite database matching applied to the identification of compounds generated by LC/TOF-MS. J Biomol Tech. 2008; 19:258-66.

38. Kelly JR, Borre Y, O'Brien C, Patterson E, El Aidy S, Deane J, et al. Transferring the blues: depression-associated gut microbiota induces neurobehavioural changes in the rat. J Psychiatr Res. 2016:82:109-18.

39. Benjamini $Y$, Hochberg $Y$. Controlling the false discovery rate: a practical and powerful approach to multiple testing. J R Stat Soc Ser B Stat Methodol. 1995;57:289-300.

\section{Publisher's Note}

Springer Nature remains neutral with regard to jurisdictional claims in published maps and institutional affiliations. 